
\title{
$\begin{array}{ll}\text { Research Square } & \begin{array}{l}\text { Preprints are preliminary reports that have not undergone peer review. } \\ \text { They should not be considered conclusive, used to inform clinical practice, }\end{array} \\ \text { or referenced by the media as validated information. }\end{array}$
}

\section{Stromaphane: A New Type of Two-dimensional Covalent Organic Framework with Subnanometric Slit-Shaped Pores}

\section{Jian-Jhih Peng}

Department of Chemistry, National Taiwan University, Taipei, Taiwan 10617

\section{Hau-Ren Yang}

Institute of Polymer Science and Engineering, National Taiwan University, Taipei, Taiwan

Chun-Wai Pai

Institute of Polymer Science and Engineering, National Taiwan University, Taipei, Taiwan

\section{Wei-Ting Chen}

Department of Molecular Science and Engineeringand Research and Development Center for Smart

Textile Technology, National Taipei University of Technology, Taipei, Taiwan

\section{Chong-You Chen}

Department of Molecular Science and Engineeringand Research and Development Center for Smart

Textile Technology, National Taipei University of Technology, Taipei, Taiwan

\section{Chang-Yun Li}

Department of Molecular Science and Engineeringand Research and Development Center for Smart Textile Technology, National Taipei University of Technology, Taipei, Taiwan

\section{Shou-Ling Huang}

Department of Chemistry, National Taiwan University, Taipei, Taiwan 10617

\section{Yen-Chen Chen}

Department of Chemistry, National Taiwan University, Taipei, Taiwan 10617

\section{Shern-Long Lee}

Department of Chemistry, National Taiwan University, Taipei, Taiwan 10617

\section{Yu-Chun Chuang}

National Synchrotron Radiation Research Center, Hsinchu, Taiwan https://orcid.org/0000-0002-28795381

\section{Hwo-Shuenn Sheu}

National Synchrotron Radiation Research Center, Hsinchu, Taiwan https://orcid.org/0000-0003-13345895

\section{I-Jui Hsu ( $\square$ ijuihsu@mail.ntut.edu.tw)}

Department of Molecular Science and Engineeringand Research and Development Center for Smart Textile Technology, National Taipei University of Technology, Taipei, Taiwan https://orcid.org/00000001-5938-5783 


\section{Chun-hsien Chen ( $\nabla$ chchhchen@ntu.edu.twh )}

Department of Chemistry, National Taiwan University, Taipei, Taiwan 10617 https://orcid.org/00000001-5507-3248

\section{Yu-Ying Lai ( $\nabla$ yuyinglai@ntu.edu.tw )}

Institute of Polymer Science and Engineering, National Taiwan University, Taipei, Taiwan https://orcid.org/0000-0002-1921-6923

\section{Guoqiao Lai ( $\square$ laiguoqiao@aliyun.com )}

5Key Laboratory of Organosilicon Chemistry and Material Technology of Ministry of Education, Hangzhou Normal University, Hangzhou, Zhejiang 311121, China

\section{Tien-Yau Luh ( $\nabla$ tyluh@ntu.edu.tw)}

Department of Chemistry, National Taiwan University, Taipei, Taiwan 10617 https://orcid.org/00000002-0415-6454

\section{Article}

Keywords: two-dimensional covalent organic framework, 2D-COF, ladderphane, stromaphane

Posted Date: June 29th, 2020

DOI: https://doi.org/10.21203/rs.3.rs-36321/v1

License: (c) (i) This work is licensed under a Creative Commons Attribution 4.0 International License. Read Full License 


\section{Abstract}

Stromaphane is a new type of two-dimensional covalent organic framework (2D-COF), constituting of multiple layers of ladderphanes where two adjacent ladderphane motifs share a common polymeric backbone. The linkers for each ladderphane moiety are perpendicular to the polymeric backbones and staggered with the linkers in the immediate neighbouring ladderphane layers. The framework of the stromaphane contains numerous slit-shaped pores with a width in subnanometres and length in nanometers. This 2D-COF is synthesized by Grubbs G-I catalyst-mediated stereospecific ring opening metathesis polymerisation of a biscyclopropene derivative having a benzene triad linker. The overall process can be considered as a self-intercalation polymerisation of a biscyclopropene. The structure of the 2D-COF has been proved by XRD measurements, DFT simulation, and STM images. This 2D-COF is composed of substituted poly(methylene-E-vinylene) (PMEV) backbones and benzene triad linkers to form a two-dimensional framework with multiply symmetrically distributed subnanometric slit-shaped pores.

\section{Main}

Research on two-dimensional covalent organic frameworks (2D-COFs) has been attracting increasing attention. ${ }^{1-8}$ Similar to the general characteristic for 2D polymers, ${ }^{9}$ those for a 2D-COF include topological planarity, crystallinity for orderly arranged repeating units, covalent bonds between recurring constituents, single molecular or atomic thickness, and free-standing integrity. Since it is crucial for 2D-COFs to be crystalline, stereochemical control in the polymerization is inevitable to synthesise 2D-COFs. Most 2DCOFs are obtained basically by functional group transformations under various conditions. ${ }^{7,8}$ Both reversible and irreversible reactions can be used for the construction of 2D-COFs. ${ }^{7,8}$ 2D-COFs with imine, aminal, boraxine or boronate, and triazine linkages have been well documented. ${ }^{1-8,10,11}$ The synthesis of fully conjugated $\mathrm{sp}^{2}$-carbon-based $2 \mathrm{D}-\mathrm{COF}$ has recently been disclosed. ${ }^{12}$ In addition, an electric-field induced reversible interconversion between a supramolecular network and a 2D-COF has been reported. ${ }^{13}$ The chemical modification of linkages in 2D-COFs has offered an alternative route for the production of new 2D-COFs with linkers that would be difficult to prepare by direct synthesis. ${ }^{14}$

The dimensions of pores in 2D-COFs can range from sub-nanometres to tens of nanometres. ${ }^{7}$ Symmetric pores with $C_{n}$ rotation axis are common in binary 2D-COFs. Pores with rectangular or similar crosssections are known in ternary 2D-COFs. There has been increasing interests in membranes having subnanometric pores for desalination. ${ }^{15}$ Graphene membrane offers an extremely cost-efficient candidate for filtration. ${ }^{16}$ The present methods uses ablation of certain carbons from graphene oxide monolayer under various conditions leading to the formation of holes. ${ }^{17}$ However, a reliable control of pore sizes is not easy. ${ }^{18}$ We now wish to report the use of self-intercalation polymerisation ${ }^{19,20}$ concept to synthesise a new type of 2D-COF-namely, stromaphane, which is defined as multiple layers of symmetric ladderphanes (Figure 1a), ${ }^{21-24}$ where the adjacent ladderphanes share a common polymeric backbone. The prefix stroma is based on the Greek word $\sigma \tau \rho \rrbracket \mu a$, which means layer. The linkers on each ladderphane motif are 
staggered with the linkers in the immediate neighbouring ladderphane layers (Figure 1a). Structurally, the framework of stromaphane constitutes of slit-shaped pores with a width in subnanometres and tunable length in nanometres.

Transition metal-catalysed polymerisations have enable diverse chemo- and/or stereo-selective syntheses of one-dimensional polymers. ${ }^{25}$ We have extensively applied ruthenium- ${ }^{26}$ or molybdenum-based catalysts $^{27}$ for the stereoselective ring opening metathesis polymerisation (ROMP) ${ }^{28}$ of N-arylpyrrolidenefused norbornene or cyclobutene derivatives (NB or CB) leading to the corresponding polymers, PNB and PCB, respectively. Double bonds in PNB are all in trans configuration, when the Grubbs G-I catalyst ${ }^{26}$ is used, ${ }^{28-30}$ and in cis form in both $\mathbf{P N B}^{31}$ and $\mathbf{P C B}^{32}$ upon treatment with a Schrock-Hoveyda catalyst SHMo (Figure 1b). ${ }^{27}$ The presence of the $\mathrm{N}$-aryl pyrrolidine moiety in NB and $\mathbf{C B}$, is vital for the stereoselective formation of the corresponding polymers PNB and PCB. The pendants in PNB and PCB thus obtained are all aligned towards a similar direction. ${ }^{29-33}$ This kind of conformation offers a unique route to synthesise both symmetric and unsymmetrical double stranded ladderphanes (Figure 1a). ${ }^{21-24}$

ROMPs of cyclopropene derivatives have been briefly explored. ${ }^{33-38}$ In a manner similar to those described above, G--catalysed ROMP of cyclopropene spirally linked with N-aryl azetidine (CP) gives 70\% yield of N-arylazetidine appended poly(methylene-vinylene) PMEV stereospecifically with all double bonds in E-configuration together with 25\% yield of the corresponding cyclohexadiene derivative CHD-1 (Figure 1c).$^{38}$ Unlike PNB and PCB mentioned above, all of the trans-double bonds in PMEV cause the Narylazetidine pendants to orient up and down in an alternating manner. It seems likely that, during the course of polymerisation of $\mathrm{CP}$, the pendant of the incoming monomeric unit might orient in the direction opposite to that of the pendant immediately next to the ruthenium carbene intermediates ERu-carb-i, where $\mathbf{i}=1,2, \ldots, \mathbf{n}$ is the number of repeating units (Figure $1 \mathrm{c}$ ). During the chain-growth process, $\boldsymbol{Z} \mathbf{R u}$-carb(i+2) may also be formed. Since the ruthenium carbene and the e,z-double bond are in close proximity, ring closure metathesis (RCM) will rapidly occur to give CHD-1 and regenerate ERu-carb-i, which will continuously undergo chain growth to yield PMEV stereospecifically with all double bonds in the $E$ configuration. ${ }^{38}$ This stereospecific synthesis of PMEV is expected to be applicable for the construction of two-dimensional stromaphane SP-1.

We have previously shown that a 3,3-dialkenylazetidine moiety would adopt syn-syn, anti-syn, or anti-anti (Figure 2) conformation. ${ }^{38}$ Since crystallinity is an important criterion for 2D-COF, the alkenyl moieties in stromaphane should adopt the same conformation throughout the polymeric scaffold. Accordingly, as shown in Figure 2, stromaphane may have three possible structures, SP-0-ss, SP-0-as, and SP-0-aa. The spacing between two adjacent $\mathrm{N}$-aryl-azetidine pendants on the same side of the polymeric backbone is estimated to be around $0.75,0.60$, and $0.45 \mathrm{~nm}$ for syn-syn, anti-syn, and anti-anti conformations, respectively.

\section{Results And Discussion}




\section{Strategy}

Intercalation polymerisation has been extensively employed for preparing layered nanocomposites. ${ }^{19,20}$ However, to the best of our knowledge, the use of this protocol to synthesise 2D polymers has not been reported. It is envisaged that a pathway similar to that shown in Figure 1c might occur, when biscyclopropene BCP-0 with a linker is employed (Figure 3a). Thus, the ROMP of BCP-0 will initially generate stereospecifically a linear oligomer/polymer (e.g., Tetra-0) that is structurally similar to PMEV. As shown in Figure 1c, the pendant of the incoming cyclopropene unit and the pendant directly attached to the ruthenium carbene intermediate as in Ru-carb-1 will be in opposite directions. Cross metathesis between these two neighbouring cyclopropene rings on the same side of the polymeric backbone in Tetra$\mathbf{0}$ is unlikely. Instead, cross metathesis of one of the cyclopropene groups in BCP-0 from the bulk with two cyclopropene rings on the same side of Tetra-0 would lead to the formation of $2 \mathrm{D}-\mathbf{0}$. The continuation of these procedures might eventually produce two-dimensional stromaphane SP-0 (Figure 3a). The overall process can be considered to be the self-intercalation polymerisation of BCP-0.

2D-COFs need rigid frameworks. ${ }^{7,8}$ The incorporation of flexible linkers into the framework may cause defects in the crystal structure, even though highly crystalline, 2D-COFs containing somewhat flexible motifs have recently been explored. ${ }^{39-4}$ As shown in Figure 2, irrespective of the conformation for alkenyl motifs in stromaphane, the separation between linkers should be in the subnanometre range. Room for the movement of linkers would therefore be fairly limited. It is envisioned that the presence of slightly flexible linker might facilitate the intercalation polymerisation.

\section{Synthesis of BCP-1}

Biscyclopropene BCP-1 was obtained in $77 \%$ yield via the $S_{N} 2$ reaction of carboxylate CP-1 $^{38}$ and a,a'dibromo- $p$-xylene (Figure $3 b$ ). The benzene triad (three benzene rings) in the linker of BCP-1 has limited flexibility to adopt the right conformation for the chain growth process.

\section{Self-intercalation polymerisation of BCP-1}

Treatment of BCP-1 with $10 \mathrm{~mol} \%$ of G-I in DCM at ambient temperature for $2 \mathrm{~h}$ afforded the corresponding crude polymer (hereafter abbreviated as CDP) in 95\% yield (Figure 3b). Immediately after workup, CDP was initially sparingly soluble in chloroform, but it gradually formed aggregates that were insoluble in any solvent. Accordingly, immediately after CDP was obtained, characterisation and microscopic examinations were performed Gel permeation chromatography (GPC) analysis of CDP shows a bimodal distribution with $M_{n}$ values around 6.0 and $12.2 \mathrm{kD}$ (Figure S1).

The ${ }^{1} \mathrm{H}$ NMR spectrum of CDP (Figure S2) exhibits two broad peaks centred at $\mathrm{d} 5.80$ and $6.10 \mathrm{ppm}$ that were assigned to the absorptions of olefinic protons attributed to PMEV backbone and cyclohexadiene fragments, respectively. The ratio was about $4.3: 1$ after deconvolution analysis of these peaks. It is worth noting that this ratio may not reflect the actual composition because of the solubility problem. These results suggest that CDP may constitute two polymeric components with one containing PMEV 
motifs and the other cyclohexadiene moieties. Another possibility would be a single species containing both PMEV and cyclohexadiene functionalities.

As will be discussed in the next two sections, the flexibility of the benzene triad linker is an impediment for the resolution of the powder XRD and STM images. Nevertheless, the long range ordered structural pattern offers a strong evidence for crystallinity feature and two-dimensional scaffold.

\section{XRD pattern and the structure of stromaphane SP-1}

Under optical microscopy, a sample of CDP was found to consist of crystalline SP-1 (Figure S3) and amorphous powder AMP-1 (Figure S4) which were separated manually. A sample of SP-1 in a $0.3 \mathrm{~mm}$ capillary tube was subjected to the XRD experiments XRD measurements were performed using beamlines TPS09A and TLS01C2 with wavelengths of $0.082656 \mathrm{~nm}$ and $0.103321 \mathrm{~nm}$, respectively. Figure $4 \mathrm{a}$ shows the one-dimensional XRD patterns obtained with $0.103321 \mathrm{~nm}$, and the corresponding two-dimensional image pattern is displayed in Figure S5. The results confirm the high crystallinity for SP1. Further processes (see Methods and Supplementary Information) resulted in having cell constants: $a=$ $6.0455(3) \mathrm{nm}, b=0.92292(4) \mathrm{nm}, c=1.15838(6) \mathrm{nm}$, and $\mathrm{b}=90.649(3)^{\circ}$ with monoclinic Pa space group and the agreement factors were $R_{w p}=1.17 \%$ and $R_{p}=0.72 \%$ (Figure S5).

As shown in Figure $4 b$, the two nitrogen atoms on the linker flanked by the benzene triad are separated by about $2.3 \mathrm{~nm}$ and the distance between two benzene rings in the triads is $0.9 \mathrm{~nm}$. The orientation of benzene rings in benzene triads are not homogeneous. The fluxional behaviour of these aromatic motifs reflects the presence of a somewhat disordered crystal structure of SP-1. The centre-to-centre distance between the benzene ring of a triad to the corresponding benzene ring of the adjacent triad is in the range of $0.44-0.56 \mathrm{~nm}$. This spacing represents the span of each monomeric unit and is also the width of the slits in SP-1. In comparison with the cartoons in Figure 2, the two geminal olefinic moieties at the $C_{3^{-}}$ position of each azetidine ring in PMEV skeletons may likely adopt anti-anticonformation. The spacing of 0.44-0.56 nm allows fairly little room for the limited movement of the triad linker. Such a minor structural disorder may not significantly affect the long-range order of crystallinity in stromaphane SP-1. In order to avoid steric congestion, the dihedral angle between the two geminal olefinic groups at the $C_{3^{-}}$ position of azetidine ring ranges from 30 to $42^{\circ}$. In addition, both the simulated XRD structure and the STM image shown in the next section revealed that the triad linker in a ladderphane stripe and the linkers in the two immediate next two ladderphane stripes are in a staggered relationship.

The length of the slit, that is the distance between two quaternary carbon atoms at both ends of the slit, is about $3.3 \mathrm{~nm}$. This spacing would approximately be the separation between two PMEV skeletons in SP-1.

\section{STM Images}

Figure 5 shows the STM images of SP-1 on highly ordered pyrolytic graphite (HOPG). The long-range ordered and striped features shown in Figure $5 a$ confirm the simulation results. Figure $5 b$ further confirms the XRD analysis results that indicate that SP-1 is somewhat rigid and 2D-patch-like polymer. The 0.45 
$\mathrm{nm}$ separation between adjacent triad linkers further suggests that olefinic moieties in the PMEV skeleton may adopt the E-configuration and anti-anti conformation.

The stripe directions are attributed to the PMEV backbones, and the angles of approximately $120^{\circ}$ between the stripes of different patches (Figure 5a) are consistent with the three-fold symmetry of the underlying HOPG. These results suggest fairly strong interactions between the SP-1 and the substrate. Figures $5 \mathrm{c}$ and $\mathrm{d}$ reveal that, in between stripes, there are other fine structures that are benzene triad linkers. As mentioned above, these linkers are somewhat fluxional, and a deviation from the alignment may occasionally occur. In addition, the bright features that may be attributed to the images of benzene triads, are significantly smaller than what was expected for the benzene rings. As shown in Figure 4b, some benzene rings are tilted from the plane of the 2D scaffold in the simulated structure for SP-1. Indeed, about $25 \%$ variation has been found in the distances between two benzene rings in adjacent linkers in this calculated structure. Figure $5 \mathrm{~d}$ is superimposed with a model calculated from the XRD results. The interactions between SP-1 and HOPG may also account for the structural discrepancy. Regardless of these minor deficiencies, the structural features shown in Figures 4 and 5 are consistent with a 2D-sheet-like structure for stromaphane SP-1.

As shown in Figure 5a and b, the crystalline morphology of SP-1 can spread over $40 \times 40 \mathrm{~nm}^{2}$ in a highly regular manner on the HOPG surface. The length of each monomeric unit is about $3.0 \mathrm{~nm}$, and the distance between two adjacent linkers is $0.45 \mathrm{~nm}$. The total number of monomeric units on this area (40 $\times 40 \mathrm{~nm}^{2}$ ) would be around 1,200 or 75 monomeric units per $100 \mathrm{~nm}^{2}$.

\section{How can cyclohexadiene be excluded from SP-1?}

In the previous sections, both the STM image (Figure 5) and XRD simulation results (Figure 4) suggest the homogeneous structural feature for SP-1. It is immensely striking to see the selective formation of SP1 with all double bonds in the trans configuration and anti-anti conformation. As shown in Figure 1c, the initial product such as Ru-carb-2 in the chain growth process adopts the trans-configuration and anticonformation for the newly formed double bond. When the product is a single stranded polymer like PCP, equilibrium may exist between Ru-carb- $\mathbf{2}_{\text {anti }}$ and Ru-carb- $\mathbf{2}_{\text {syn }}$ or the like and the latter with the synconformation may be thermodynamically preferred. However, this kind of equilibrium might not be particularly favourable for the growth of more rigid 2D-framework of SP-1. In other words, the initially formed anti conformation would continuously generate the corresponding anti conformation throughout the polymerisaton process.

During the course of polymerisation, the intermediate species having a Z-double bond might be theoretically generated along with those with the E-double bond. However, the homogeneous nature of SP-1 suggests that the $Z$-double bond might not be incorporated in the polymeric scaffold. If the $Z$-double bond were formed and the fragment containing such double bond were linked to the 2D-framework (Figure 6a), the ruthenium carbene moiety (2D-Z-Ru-1) would be trapped into the sub-nanometric gap between the two adjacent pendants. It is known that tricyclohexylphosphine ligand remains ligating to 
ruthenium in the ruthenium-carbene-catalysed metathesis process. ${ }^{26}$ The spacing allowed for ruthenium carbene in 2D-Z-Ru-1 might not be sufficient to contain this bulky ligand.

In a similar manner, the tricyclohexylphosphine ligand would not fit into the aperture between the two adjacent pendants in the possible cyclohexadiene-comprising intermediate 2D-CHD-Ru-1. It seems unlikely that the ruthenium carbene species in intermediates similar to $\mathbf{2 D - Z - R u - 2}$ would continue catalysing the chain growth process because of steric congestions. If the positions of Ru-carbene and the Z-double bond are swapped such that the Ru moiety is at the exo position as in 2D-Z-Ru-exo-1 (Figure 6c), severe strain will exist around the $Z$-double bond.

When ROMP takes place between 2D-E-Ru- 2 and a biscyclopropene (such as BCP-1), the initially formed 2D-Z-Ru-2 with the Z-double bond would have an unreacted cyclopropene group at the end of the linker. This fragment would be movable to generate a space to house the tricyclohexylphosphine ligand as in 2D-Z-Ru-2. After ring closure metathesis, the cyclohexadiene-containing fragment CHD-2 would be liberated from the framework to regenerate 2D-E-Ru-2 that would continue the chain growth process leading to SP-1 (Figure 6b). These hypotheses reasonably explain why the cyclohexadiene moiety was absent in SP-1.

\section{Conclusion}

We have demonstrated the design, synthesis, and characterisation of a new type of 2D-COF stromaphane SP-1. The uniqueness of this approach includes the first use of a transition metal catalyst to synthesize a 2D-COF. Under G-I-catalysed ROMP conditions, the cyclopropene derivative CP enables the stereospecific

synthesis of substituted PMEV, while the pendants are arranged up and down in an alternating manner. ${ }^{38}$ Based on this preliminary result, a new building block, namely, bis-cyclopropene BCP-1 with a somewhat flexible benzene triad linker connected to azetidine moiety, has been synthesized. This unique monomer has paved the way to the discovery of self-intercalation polymerisation to synthesize a new type of 2DCOF, stromaphane SP-1, by Grubbs G-I-catalysed ROMP of BCP-1. The PMEV skeletons with the benzene triad linkers form the basis of the framework for the fairly compact SP-1 The subnanometric slit-shaped pores of SP-1 are symmetrically and homogeneously arranged throughout the planar scaffold. The pore widths were measured and found to be $0.45 \mathrm{~nm}$, whereas the pore length varied depending on the length of the linker. This circumscribed facet of SP-1 stands out as a unique 2D-COF. Although linkers in SP-1 could be fairly flexible, but, as mentioned above, the surroundings offer little space for the fluxional movement of the linkers. As such, although local defects are present, the long-range-ordered and homogeneous structural features of SP-1 can be clearly seen by XRD and STM examinations. The structural information obtained from XRD and STM image suggests that SP-1 is homogeneous, and that the cyclohexadiene moiety might not be present in SP-1. The proposed mechanism shown in Figure 6 may rationalize why cyclohexadiene is not incorporated in SP-1. The fate of the cyclohexadiene motif, however, remains to be clarified. 
The modification of linkers might enable the manipulation of the slit length, and the thus-obtained stromaphane could serve as a potential membrane having homogeneously distributed subnanometric pores for various applications. If the linker is an optoelectronic active oligoaryl moiety, a similar number of chromophores would be well aligned. The behaviour of well-oriented chromophores in these 2D-COF materials for optoelectronic applications might be different from those of small molecules having the same chromophores, but packed in amorphous manner. The present study has thus laid the foundation for further investigations.

\section{Methods}

\section{XRD determination and simulation of the structure for SP-1}

The powder x-ray diffraction experiments were carried out using beamlines TLS01C2 and TPS09A at National Synchrotron Radiation Research Center (NSRRC) in Taiwan. A crystalline sample of SP-1 was placed in a $0.3 \mathrm{~mm}$ capillary and the sample was spinning during the course of measurements. The wavelengths used for data collections were $0.082656 \mathrm{~nm}$ and $0.103321 \mathrm{~nm}$ at beamlines TPS09A and TLS01C2, respectively Figure 4a shows the 1-D XRD pattern measured at $0.103321 \mathrm{~nm}$, and the corresponding 2-D image pattern is displayed in (Figure S5). The XRD pattern indicates the high crystallinity for SP-1, but attempts to collect data by single crystal diffractometer were unsuccessful due to ring diffraction patterns which implied that the quality of the crystal was not good enough. The diffraction peaks of 1-D XRD pattern was initially deconvoluted by profile fitting and the diffraction angles were indexed by DICVOL04 program. ${ }^{42}$ The crystal may adopt monoclinic system with cell constants : $a=6.038(2) \mathrm{nm}, b=0.9233(4) \mathrm{nm}, c=1.1573(4) \mathrm{nm}, \mathrm{b}=90.59(3)^{\circ}$, and the possible space group is $\mathrm{Pa}$ which was checked by CMPR program. ${ }^{43}$ The cell constants were ensured that all input from 73 reflections $\left(2 q=1.90^{\circ} \sim 22.23^{\circ}\right)$ can be indexed and the differences of $2 q$ at each reflection are smaller than $0.03^{\circ}$. The figures of merit of $F(20)$ and $F(73)$ are 41.6 and 28.0, respectively. The indexing results are listed in supplementary information. The results were further refined by Le Bail profile fitting method in GSAS program. ${ }^{44-46}$ The agreement factor of $R_{w p}=1.17 \%$ and $R_{p}=0.72 \%$, and cell constants are: $a=6.0455(3) \mathrm{nm}, b=0.92292(4) \mathrm{nm}, c=1.15838(6) \mathrm{nm}, \mathrm{b}=90.649(3)^{\circ}$. An attempt to do ab initio structure determination in real space by global optimization methods was unsuccessful. The use of crystal structure simulation by periodic-DFT method in CRYSTAL17 program based on the experimental cell constants was pursued. All detailed information about the DFT calculation and the geometry of fractional coordinates in the format of crystallographic information file (CIF) is summarised in supplementary information. The simulated XRD pattern based on the DFT optimized structure and the experimental XRD data with Le Bail refinement results are displayed in Figure S6.

\section{STM imaging}

STM imaging was performed with a NanoScopellla controller (Veeco Metrology Group/Digital Instruments). A sample of an aliquot of $10 \mu \mathrm{L}$ of SP-1 in phenyloctane was placed on HOPG (Advanced 
Ceramics, ZYH grade) under ambient conditions. ${ }^{22,47}$ The STM tips were mechanically cut from Pt/Ir wires (California Fine Wires). The images were essentially raw data with a first-order flatten to minimise noise and presented without further processing.

\section{Declarations}

\section{Acknowledgments}

This work was supported by the Ministry of Science and Technology, Taiwan, and by the National Taiwan University. We are grateful to the National Center for High-Performance Computing for computer time and facilities. IJ.H. thanks Research and Development Center for Smart TextileTechnology of National Taipei University of Technology for partial supporting this work. Thanks are also due to Dr. Jey-Jau Lee and Mr. Bo-Hao Chen of NSRRC for assistance and helpful discussio

\section{Contributions}

With the participation by Y.-Y.L. and G.-Q.L., T.-Y.L. conceived and designed the project. J.-J.P. executed the synthesis of stromaphane and related polymers. C.-h.C. and his coworkers (S.-L.L. and Y.-C.C.) did the STM image of stromaphane. I.-J.H. and his students (W.-T.C., C.-Y.C., and C.-Y.L.) collaborated with Y.-C.C and H.S.S. of NSSRC to pursue small angle X-ray diffraction studies and did the data analyses and structural simulation. Y.-Y.L. and his group members (H.-R.Y. and C.-W.P.) carried out all quantum chemistry calculations (DFT) on conformation at different stages. S.-L.H. carried out all NMR studies and analyses. All authors interpreted the data and participated in discussion and preparation of the manuscript.

\section{Ethics declarations}

Competing interests

The authors declare no competing interests.

\section{Supplementary Information}

Experimental procedures for the synthesis small molecules and stromaphane SP-1 and NMR spectra of all new compounds. GPC profiles, XRD details and the simulation of structure of stromaphanes SP-1.

\section{References}

1. Spitler, E. L. \& Dichtel, W. R. Lewis acid-catalysed formation of two-dimensional phthalocyanine covalent organic frameworks. Chem. 2, 672-677 (2010).

2. Feng, X. Ding, X. \& Jiang, D. Covalent organic frameworks. Soc Rev 41, 6010-6022 (2012). 
3. Ding, S.-Y. \& Wang, W. Covalent organic frameworks (COFs): from design to applications. Soc. Rev. 42, 548-568 (2013).

4. Waller, P. J. Gandara, G. \& Yaghi, O. M. Chemistry of covalent organic frameworks. Chem. Res. 48, 3053-3063 (2015).

5. Diercks, C. S. \& Yaghi, O. M. The atom, the molecule, and the covalent organic framework. Science 355, eaal1585 (2017).

6. Jin, Y. Hu, Y. \& Zhang, W. Tessellated multiporous two-dimensional covalent organic frameworks. Rev. Chem. 1, 0056 (2017).

7. Jiang, D. et al. Covalent organic frameworks: chemical approaches to designer structures and built-in functions. Chem. Int. Ed. Doi:10.1002/anie.201904291 (2019).

8. Lyle, S. J. Walker P. \& Yaghi, O. M. Covalent organic frameworks: organic chemistry extended into two and three dimensions. Trends Chem. Doi:10.1016/j.trechem.2019.03 001 (2019).

9. Payamyar, King, B. T. Öttinger, H. C. \& Schlüter, A, D. Two-dimensional polymers: concepts and perspectives. Chem. Commun. 52, 18-54 (2016).

10. Côté, A. et al. Porous, crystalline, covalent organic frameworks. Science 2005 310, 1166-1170 (200ㅁ).

11. Liu, J. et al. Solution synthesis of semiconducting two-dimensional polymer via trimerization of carbonitrile. Am. Chem. Soc. 139, 11666-11669 (2017).

12. Jin, E. et al. Two-dimensional $\mathrm{sp}^{2}$ carbon-conjugated covalent organic frameworks. Science 357, 673676 (2017).

13. Cai, Z.-F. Electric-field-mediated reversible transformation between supramolecular networks and covalent organic frameworks. Am. Chem. Soc. 141, 11404-11408 (2019).

14. Walker, P. J. et al. Chemical conversion of linkages in covalent organic frameworks. Am. Chem. Soc. 138, 15519-15522 (2016).

15. Cohen-Tanugi, D. \& Grossman, J. C. Water desalination across nanoporous graphene. Nano Lett. 12, 3602-3608 (2012).

16. Surwade, S. P. et al. Water desalination using nanoporous single-layer graphene. Nat. Nanotechnol. $2015,10,459-464$.

17. Lin, L.-C. \& Grossman, J. C. Atomistic understandings of reduced graphene oxide as an ultrathin-film nanoporous membrane for separations. Commun. 6, 8335- (2015).

18. Raffone, F. Savazzi, F. Cicero, G. Controlled pore generation in single layer graphene oxide for membrane desalination. Phys. Chem. Lett. 10, 7492-7497 (2019).

19. Guo, Y. et al. Intercalation polymerization approach for preparing graphene/polymer composites. Polymers 10, 61/1-61.2 (2018).

20. Duguet, E. Ray, S. \& Robles, J. M. M. Intercalation polymerization In Encyclopedia of Polymer Science and Technology, $4^{\text {th }}$, Ed Mark, H. F Vol 7, pp 71-92 (2014).

21. Yang, $\mathrm{H} .-\mathrm{C}$ et al. Molecular architecture towards helical double stranded polymers. Chem. Int. Ed. 45, 726-730 (2006). 
22. Chou, -M. et al. Polymeric ladderphanes. J. Am. Chem. Soc. 131, 12579-12585 (2009).

23. Luh, T.-Y. Ladderphanes-a new type of duplex polymers. Chem. Res. 46, 378-389 (2013).

24. Luh, T.-Y. \& Ding, L. Recent advances in chemistry of ladderphanes and related polymers. Tetrahedron 73, 6487-6513 (2017).

25. Hiyama, T., Ed., Comprehensive Organometallic Chemistry IIF, Vol 11, Elsevier, 2007.

26. Grubbs, R. H. \& Khosravi, E, Eds Handbook of Metathesis, $2^{\text {nd }}$, Vol 3, Wiley-VCH; (2015).

27. Schrock, R. \& Hoveyda, A. H. Molybdenum and tungsten imido alkylidene complexes as efficient olefin metathesis catalysts. Angew. Chem. Int. Ed. 42, 4592-4633 (2003).

28. Sattigeri, J. A. et al. Remarkable enhancement of second-order nonlinear optical properties of polynorbornenes having pendant chromophores use of hyper-Rayleigh scattering to estimate the tacticity of rigid rod polymers. Am. Chem. Soc. 121, 1607-1608 (2009).

29. Lin, W.-Y. et al. On the rigidity of polynorbornenes with dipolar pendant groups. Eur. J. 12, 324-330 (2006).

30. Lin, W.-Y. et al. On the tacticity of polynorbornenes with 5,6-endo pending groups having substituted aryl chromophores. Asian J. 2, 764-774 (2007).

31. Zhu, L. et al. Cis, isotactic selective ROMP of norbornenes fused with n-arylpyrrolidines Double stranded polynorbornene-based ladderphanes with Z-double bonds. Macromolecules 45, 8166-8171 (2012).

32. Lin, N.-T. et al. On the stereoselectivity of ring opening metathesis polymerization of N-arylpyrrolidinefused cyclobutenes with molybdenum- and ruthenium-alkylidene catalysts. Macromolecules 46, 7173-7179 (2013).

33. Nguyen, S. T. Johnson, L. K. Grubbs, R. H. Ziller, J. W. Ring-opening metathesis polymerization (ROMP) of norbornene by a group VIII carbene complex in protic media. Am. Chem. Soc.114, 39743975 (1992).

34. Flook, M. M. Gerber, L. C. H. Debelouchina, G. T. Schrock, R. R.Z-selective and syndioselective ringopening metathesis polymerization initiated (ROMP) by monoaryloxidepyrrolide (MAP) catalysts. Macromolecules 43, 7515-7522 (2010).

35. Dumas, A. et al. A versatile and highly Z-selective olefin metathesis ruthenium catalyst based on a readily accessible N-heterocyclic carbene. ACS Catal. 8, 3257-3262 (2018).

36. Binder, W. H. et al. Monitoring block-copolymer crossover-chemistry in ROMP: catalyst evaluation via mass spetrometry (MALDI). Macromolecules 42, 9457-9466 (2009).

37. Elling, B. \& Xia, Y. Living alternating ring-opening metathesis polymerization based on single monomer additions. J. Am. Chem. Soc. 137, $9922-9926$ (2015).

38. Peng, J.-J. et al. Stereospecific synthesis of poly (methylene-e-vinylene) by ring opening metathesis polymerization (ROMP) of substituted cyclopropene using Grubbs catalysts. Macromolecules 52, 7749-7755 (2019). 
39. Xu, L. et al. Highly crystalline covalent organic frameworks from flexible building blocks. Commun. 52, 4706-4709 (2016).

40. Zou, L. et al. Flexible monomer-based covalent organic frameworks: design, structure and function. 19, 4868-4871, (2017).

41. Sasmal, H. et al. Superprotonic conductivity in flexible porous covalent organic framework Angew. Chem. Int. Ed. 57, 10894-10898 (2018).

42. Boultif, A. Louer, D. Powder pattern indexing with the dichotomy method. Appl. Cryst. 37, 724-731 (2004).

43. Toby, B. CMPR-a powder diffraction toolkit. J. App./ Cryst 38, 1040-1041 (2005)

44. Le Bail, A. Whole powder pattern decomposition methods and applications: A retrospection. Powder 20, 316-326 (2005).

45. Larson, A. C. \& Von Dreele, R. General structure analysis system (GSAS). Tech. Rep. LAUR 86-748 Los Alamos National Laboratory (LANL) (2004)

46. Toby, B. EXPGUI, a graphical user interface for GSAS. J. Appl. Cryst. 34, 210-213 (2001).

47. Lee, S.-L. et al Shear-induced long-range uniaxial assembly of polyaromatic monolayers at molecular resolution. Am. Chem. Soc. 130, 10454-10455 (2008).

\section{Figures}




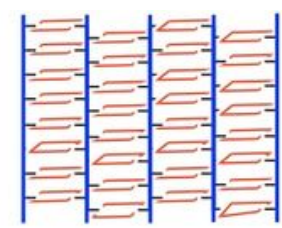

Symmetric Unsymmetric ladderphane ladderphane

Stromaphane
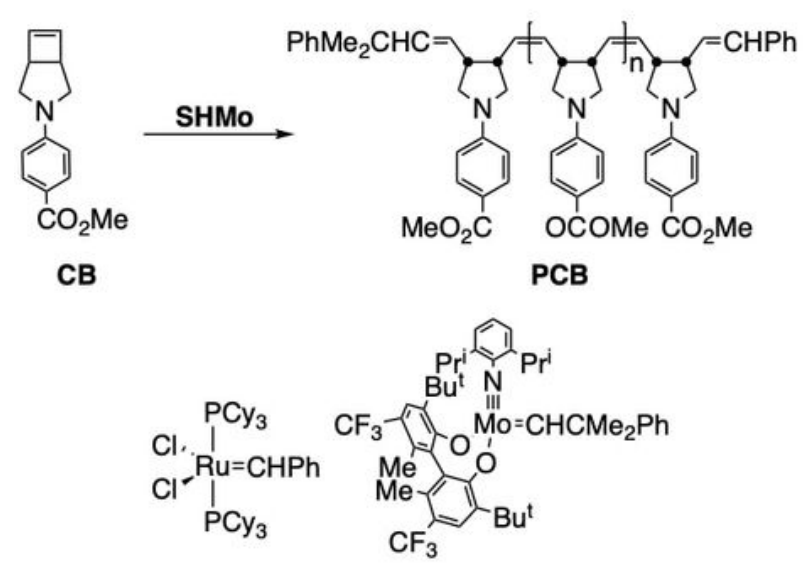

G-1

SHMo

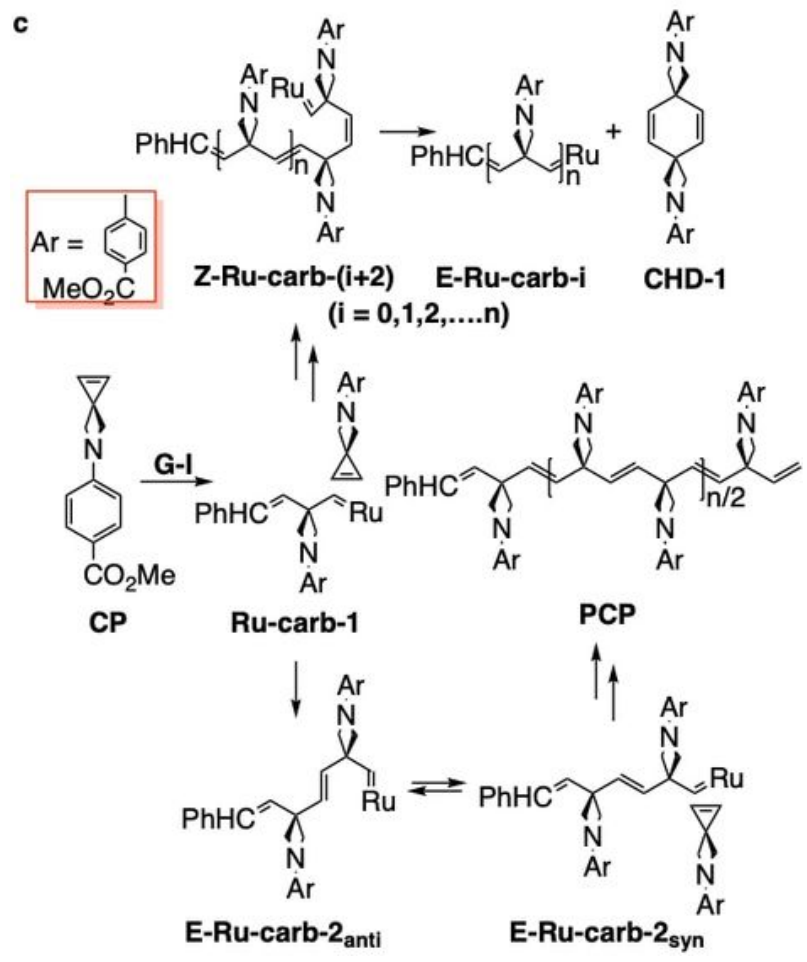

\section{Figure 1}

(a) Cartoon representation of symmetric,24-27 unsymmetrical ladderphanes,28 and stromaphane. The linkers are coloured in red. (b) G-I- or SHMo-catalysed ROMP of norbornene (NB) or cyclobutene (CB) derivatives giving isotactic PNB or PCB stereoselectively. (c) G-I-catalysed ROMP of cyclopropene derivative giving cyclohexadiene derivative (CHD-1) and substituted PMEV stereospecifically. Possible intermediates leading to PMEV are also shown in the scheme. 


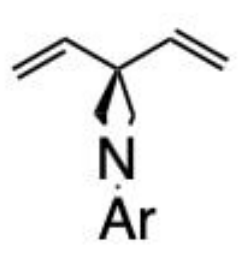

syn-syn

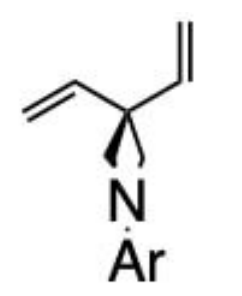

anti-syn
Ar

anti-anti

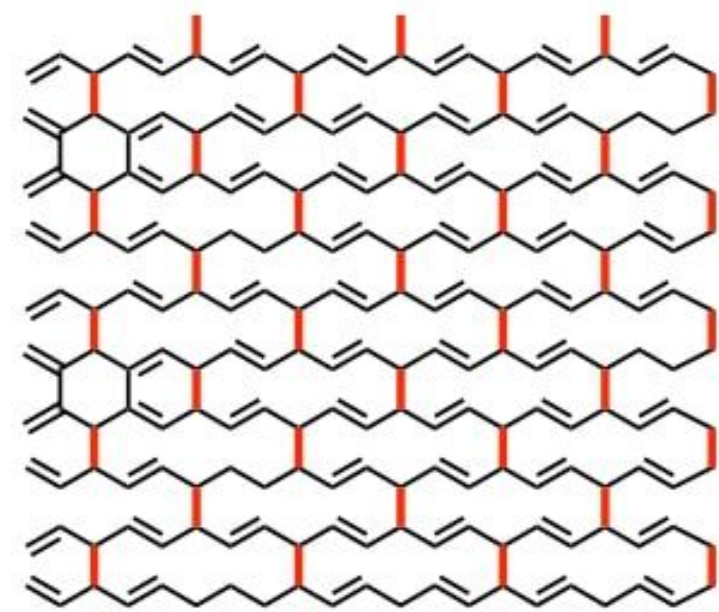

SP-0-ss
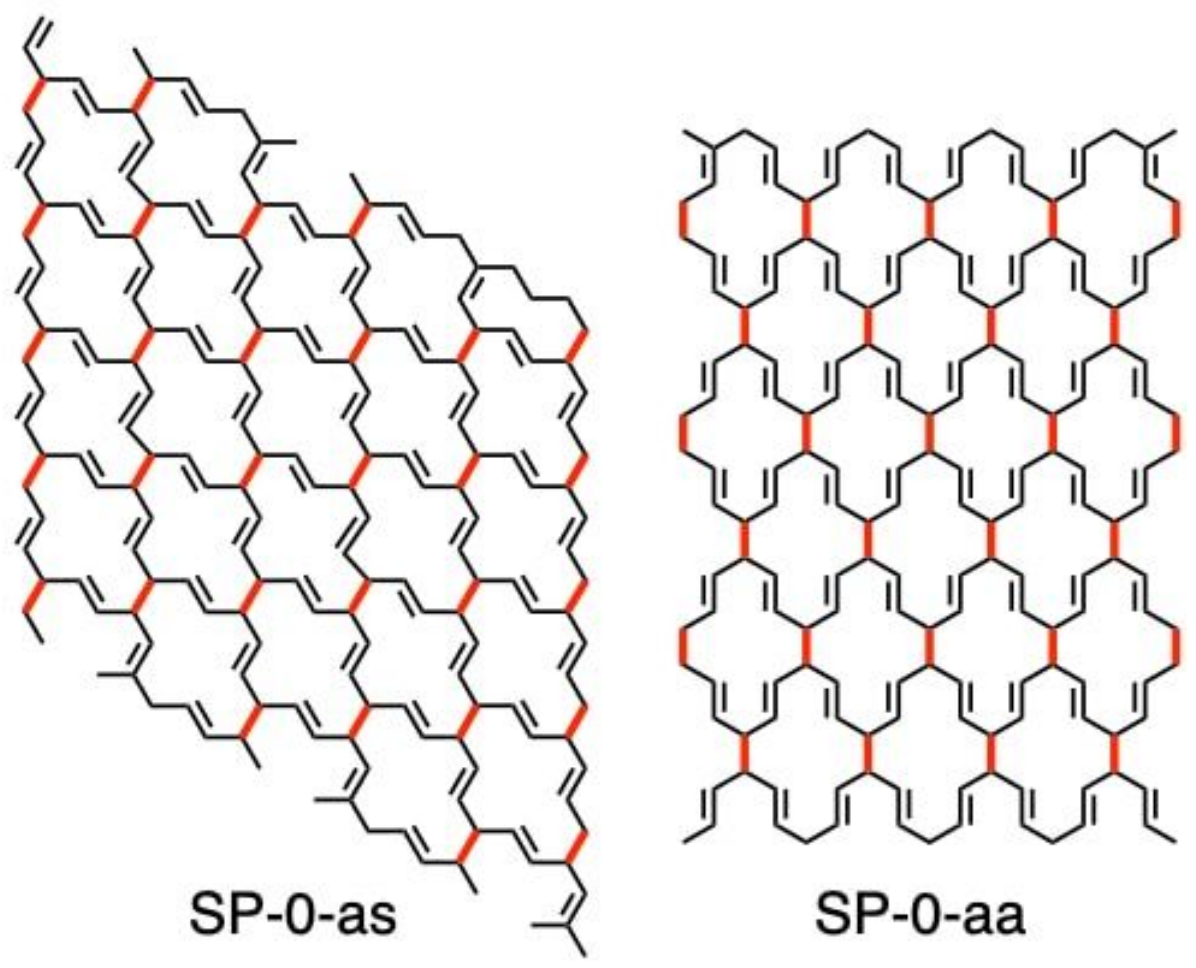

Figure 2

Three conformations (syn-syn, anti-syn, and anti-anti) of N-aryl-3,3-dialkenylazetidines and cartoon representation on the structures of stromaphanes with different conformations of dialkenylazetidine (SP0 -ss, SP-0-as, and SP-0-aa). The linkers are coloured in red. 
a

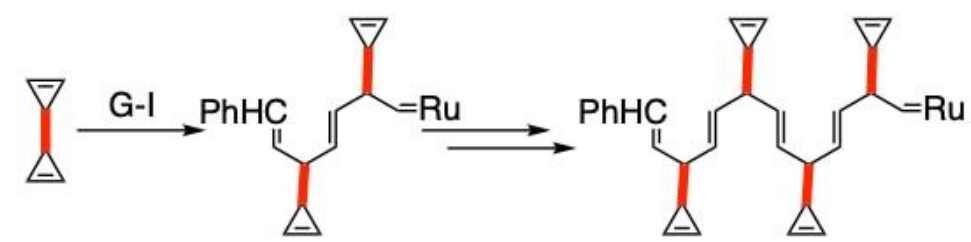

BCP-0

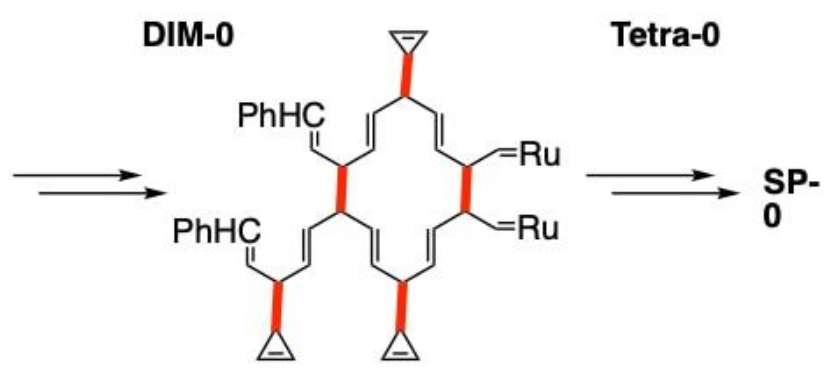

b

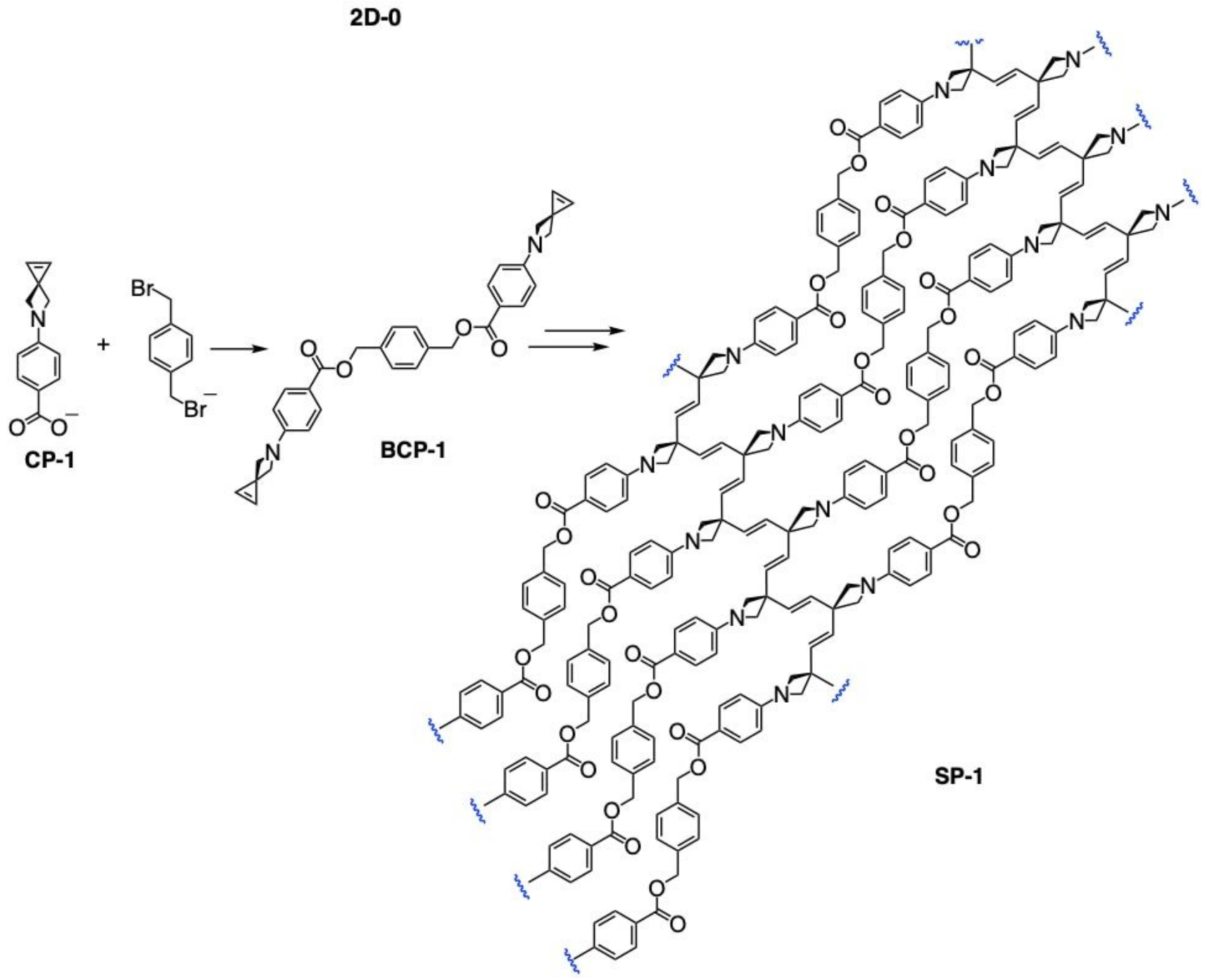

Figure 3

(a) Cartoon representation on the strategy to synthesise stromaphane (SP-0) by self-intercalation polymerisation of biscyclopropene (BCP-0). The linkers are coloured in red. (b) Synthesis of biscyclopropene derivative BCP-1 and G-l-catalysed ROMP of BCP-1 giving the corresponding stromaphane SP-1. 

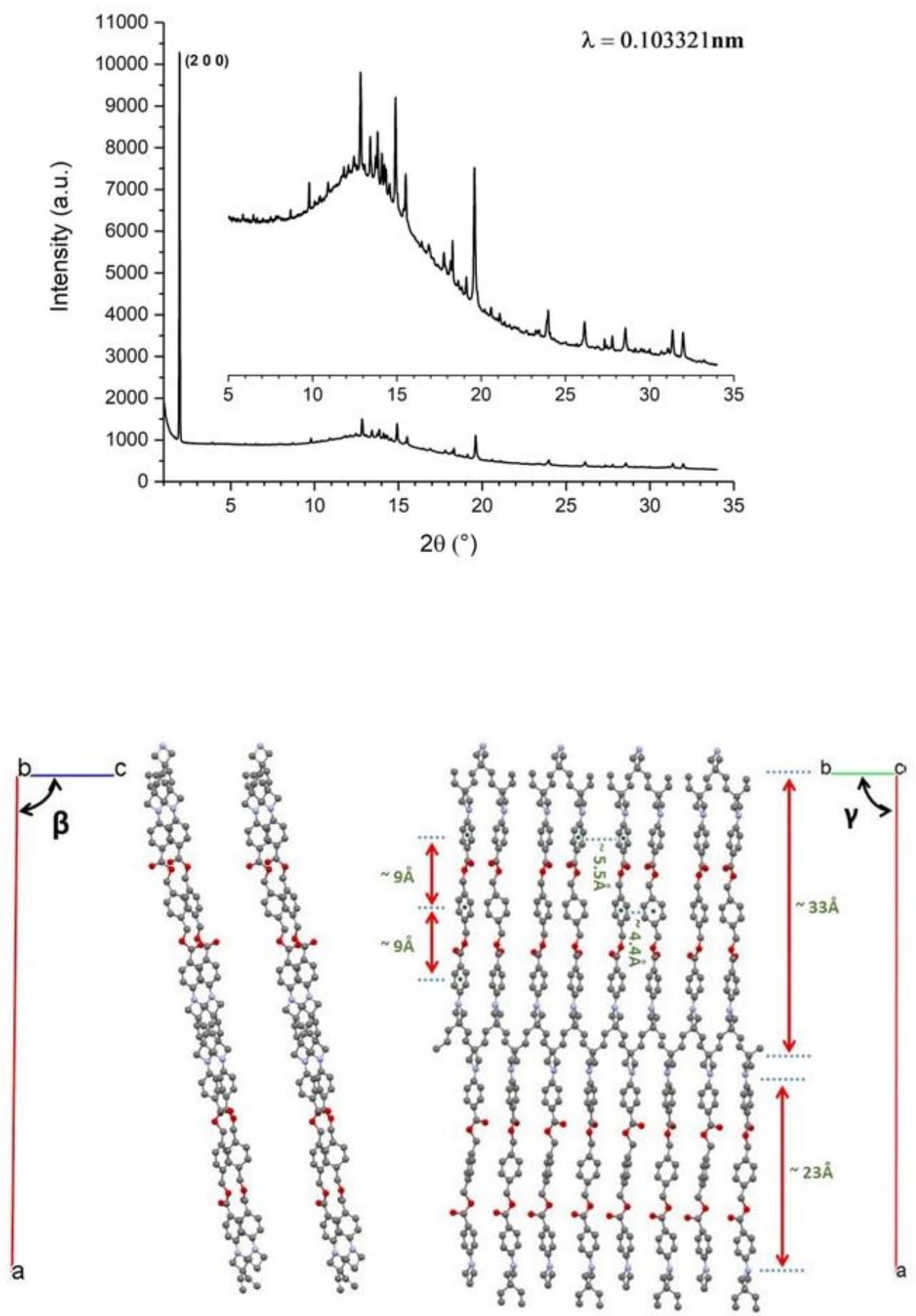

Figure 4

(a) Experimental XRD pattern. The sharp diffraction peaks indicate the long range ordering and crystallinity of SP-1. The first and strongest diffraction peak is indexed as $\left(\begin{array}{lll}2 & 0 & 0\end{array}\right)$ based on the obtained cell constants and space group (see context). (b) The packing diagram of DFT optimized crystal structure viewed along b-axis (left) and along c-axis (right). All H-atoms are skipped for clarity. The fluxional behavior of the benzene triad linker indicates the flexibility and may present somewhat disordered crystal 
structure, so that the b-axis $(\sim 0.92 \mathrm{~nm})$ and a-axis $(\sim 6 \mathrm{~mm})$ are about double the length of estimated monomer moiety adopted in anti-anti conformation ( $\sim 0.45 \mathrm{~nm}$ and $\sim 3 \mathrm{~nm}$ shown in STM results). In each benzene triad linker, the distances between two nitrogen atoms and two benzene rings are $\sim 2.3 \mathrm{~nm}$ and $\sim 0.9 \mathrm{~nm}$, respectively. The distances of benzene centroids between adjacent triad linkers are about $0.44 \mathrm{~nm}$ to $0.56 \mathrm{~nm}$. Such two dimensional long range ordering makes a 2-D ladder like structure with a slit length about $3 \mathrm{~nm}$.
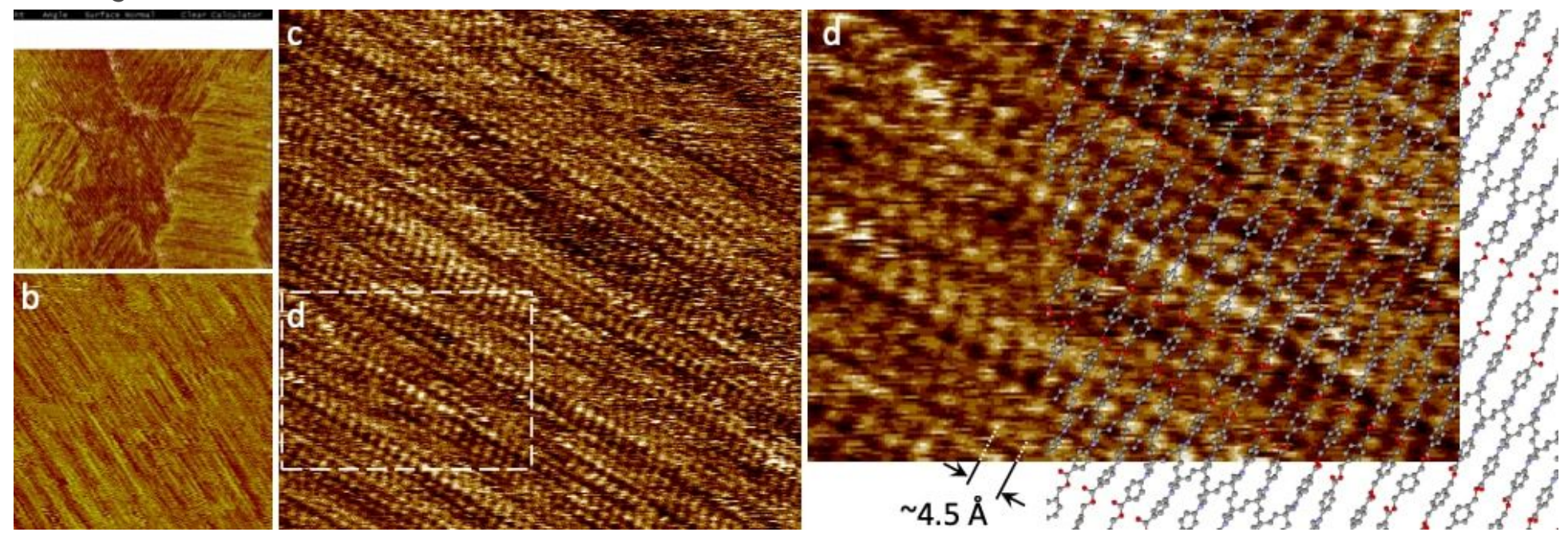

\section{Figure 5}

a and b, the crystalline morphology of SP-1 can spread over $40 \times 40 \mathrm{~nm} 2$ in a highly regular manner on the HOPG surface. The length of each monomeric unit is about $3.0 \mathrm{~nm}$, and the distance between two adjacent linkers is $0.45 \mathrm{~nm}$. The total number of monomeric units on this area $(40 \times 40 \mathrm{~nm} 2)$ would be around 1,200 or 75 monomeric units per $100 \mathrm{~nm} 2$. 
a

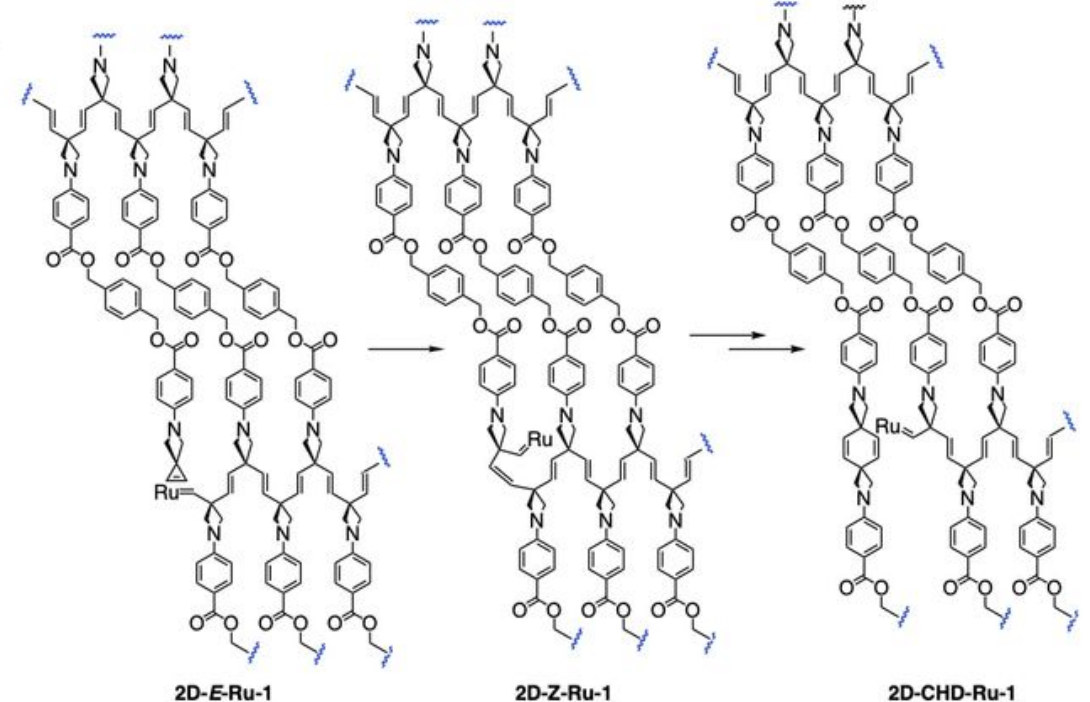

b
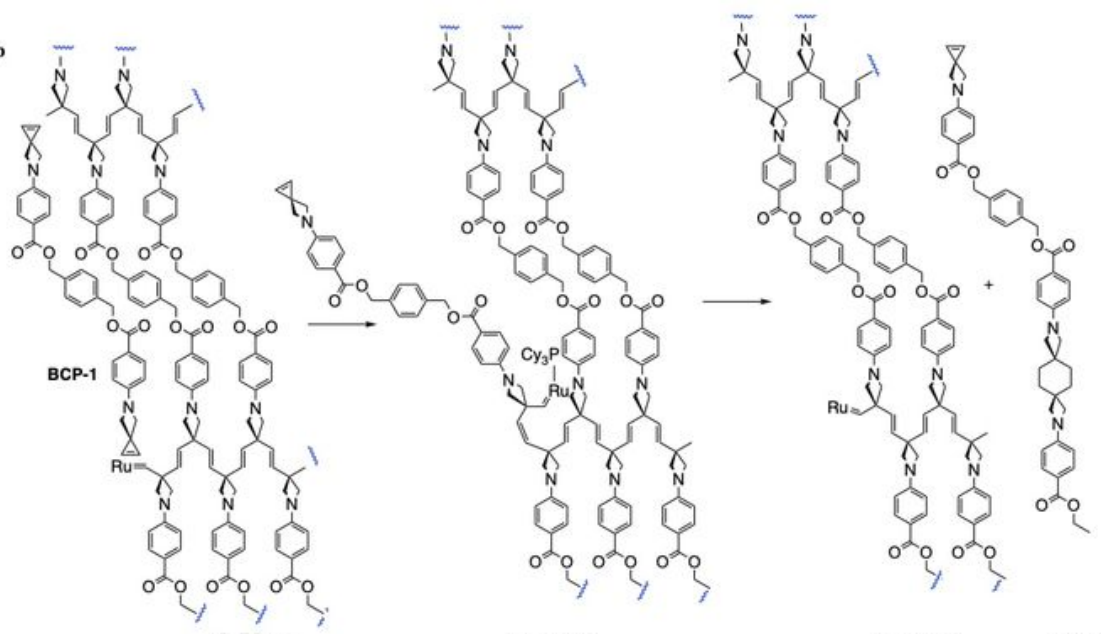

2D-Z-Ru-2

c

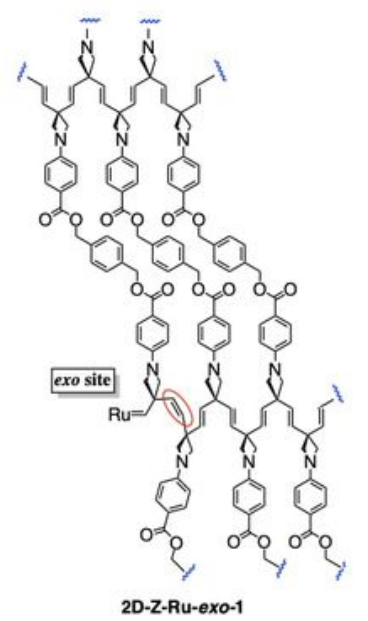

2D-E-Ru-2 CHD-2

\section{Figure 6}

(a) Possible mechanism I to rationalize why the cyclohexadiene moiety is not incorporated to SP-1. (b) Possible mechanism II to rationalize why the cyclohexadiene moiety is not incorporated to SP-1. (c) Possible intermediate 2D-Z-Ru-exo-1 with Z-double bond at endo and ruthenium carbene at the exo position. 


\section{Supplementary Files}

This is a list of supplementary files associated with this preprint. Click to download.

- SILuhr1.pdf 